\title{
Dihedral-Angle Dependence of Intermolecular Transfer Integrals in BEDT-BDT-Based Radical-Cation Salts with $\theta$-Type Molecular Arrangements
}

\author{
Tomofumi Kadoya ${ }^{1, *}$, Shiori Sugiura ${ }^{2}$, Toshiki Higashino ${ }^{3}$, Keishiro Tahara ${ }^{1}$, Kazuya Kubo ${ }^{1}$, \\ Takahiko Sasaki $^{2}$ D, Kazuo Takimiya ${ }^{4,5}$ and Jun-ichi Yamada ${ }^{1}$
}

1 Department of Material Science, Graduate School of Science, University of Hyogo, 3-2-1 Kouto, Kamigori-cho, Ako-gun, Hyogo 678-1297, Japan; taharak@sci.u-hyogo.ac.jp (K.T.); kubo@sci.u-hyogo.ac.jp (K.K.); yamada@sci.u-hyogo.ac.jp (J.-i.Y.)

2 Institute for Materials Research, Tohoku University, 2-1-1 Katahira, Aoba-ku, Sendai, Miyagi 980-8577, Japan; s.sugiura@imr.tohoku.ac.jp (S.S.); takahiko@imr.tohoku.ac.jp (T.S.)

3 National Institute of Advanced Industrial Science and Technology (AIST), Tsukuba, Ibaraki 305-8565, Japan; t-higashino@aist.go.jp

4 RIKEN CEMS, 2-1 Hirosawa, Wako, Saitama 351-0198, Japan; takimiya@riken.jp

5 Graduate School of Science, Tohoku University, 6-3 Aramaki Aza-Aoba, Aoba-ku, Sendai, Miyagi 980-8578, Japan

* Correspondence: kadoya.t@sci.u-hyogo.ac.jp; Tel.: +81-(791)-58-0164

check for updates

Citation: Kadoya, T.; Sugiura, S.; Higashino, T.; Tahara, K.; Kubo, K.; Sasaki, T.; Takimiya, K.; Yamada, J.-i. Dihedral-Angle Dependence of Intermolecular Transfer Integrals in BEDT-BDT-Based Radical-Cation Salts with $\theta$-Type Molecular Arrangements. Crystals 2021, 11, 868. https://doi.org/10.3390/cryst11080868

Academic Editor: Toshio Naito

Received: 30 June 2021

Accepted: 20 July 2021

Published: 27 July 2021

Publisher's Note: MDPI stays neutral with regard to jurisdictional claims in published maps and institutional affiliations.

Copyright: (c) 2021 by the authors. Licensee MDPI, Basel, Switzerland. This article is an open access article distributed under the terms and conditions of the Creative Commons Attribution (CC BY) license (https:// creativecommons.org/licenses/by/ $4.0 /)$.
Abstract: We report the structural and physical properties of a new organic Mott insulator (BEDTBDT)AsF 6 (BEDT-BDT: benzo[1,2-g:4,5-g']bis(thieno[2,3-b][1,4dithiin). This AsF $_{6}$ salt has the same structure as the $\mathrm{PF}_{6}$ salt. Although the anions are disordered, the donor molecules form a $\theta$-type arrangement. The temperature dependence of the resistivity exhibits semiconducting behavior. The static magnetic susceptibility follows Curie-Weiss law over a wide temperature range; however, below $25 \mathrm{~K}$, the magnetic susceptibility is in agreement with a one-dimensional chain model with the exchange coupling $J=7.4 \mathrm{~K}$. No structural phase transition was observed down to $93 \mathrm{~K}$. At $270 \mathrm{~K}$, the Fermi surface calculated by the tight-binding approximation is a two-dimensional cylinder; however, it is significantly distorted at $93 \mathrm{~K}$. This is because the dihedral angles between the BEDT-BDT molecules become larger due to lattice shrinkage at low temperatures, which results in a smaller transfer integral $\left(t_{1}\right)$ along the stack direction. This slight change in the dihedral angle gives rise to a significant change in the electronic structure of the $\mathrm{AsF}_{6}$ salt. Radical-cation salts using BEDT-BDT, in which the highest occupied molecular orbital does not have a dominant sign throughout the molecule, are sensitive to slight differences in the overlap between the molecules, and their electronic structures are more variable than those of conventional $\theta$-type conductors.

Keywords: organic conductors; organic semiconductors; molecular orbitals

\section{Introduction}

Molecular conductors have a rich phase diagram, including metallic, charge-densitywave, charge-ordering, spin-Peierls, spin-density-wave, and superconducting states [1]. In these organic conductors, the transfer integrals have a significant influence on the electronic characteristics. This occurs because the shapes of the molecular orbitals and the way they overlap determine the energy bandwidth and dimensionality of the electronic structure [2,3]. Generally, molecular conductors that have a one-dimensional (1D) electronic structure are prone to Peierls transition, which distorts their periodic structure [4]. Therefore, it is necessary to form a two-dimensional (2D) electronic structure to obtain a particular electronic state, such as superconductivity or a spin-liquid phase. The most successful example of this molecular strategy is bis(ethylenedithio)tetrathiafulvalene (BEDT-TTF), which is shown in Scheme 1a [5,6]. Due to the sulfur atoms in the ethylenedithio group, the 
molecular orbitals are expected to overlap not only in the stack direction but also between the stacks. The strain at the ethylene site at the end of the molecule prevents monotonic stacking. Thus, radical-cation (RC) salts using BEDT-TTF form $>200$ types of molecular conductors and realize a wide variety of electronic states [2]. Based on the success of BEDT-TTF, donor molecules with tetrathiafulvalene (TTF) and tetrathiapentalene (TTP) skeletons are frequently used in organic conductors, especially in RC salts [6]. The highest occupied molecular orbitals (HOMOs) in these molecules are largely localized on the sulfur atoms in the 1,3-dithiol-2-ylidene (DY) unit. Therefore, TTF and TTP derivatives have very similar HOMO shapes $[5,7]$.

(a)<smiles></smiles><smiles></smiles><smiles>C1=CSC(=C2SC3=C(S2)SC(=C2SC=CS2)S3)S1</smiles>

TTP
BEDT-TTF<smiles>c1ccsc1</smiles>

1,3-dithiol-2-ylidene (b)

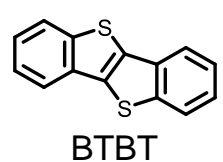

BTBT

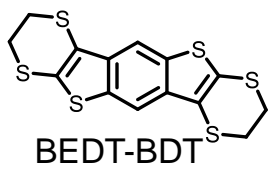

तो

Benzothiophene moiety

Scheme 1. Structural formula of (a) DY- and (b) benzothiophene-based electron donors.

The molecular orbitals of organic materials are unique to each molecule; therefore, theoretically, there are nearly infinite options. However, in the RC salts studied to date, only a limited number of donor molecules-including the DY moiety-have been used frequently, and few research examples focus on the shape and symmetry of the molecular orbital. In other words, very few examples use new molecular skeletons, which are essential for the future development of organic conductors. To address this issue, we have been developing RC salts using non-TTF molecules. We have developed molecular conductors using benzothioenobenzothiophene (BTBT), which is the basic skeleton of an excellent p-type semiconductor, for example, (BTBT) ${ }_{2} \mathrm{XF}_{6}(\mathrm{X}=\mathrm{P}, \mathrm{As}, \mathrm{Sb}$, and Ta) [8-11]. They exhibit a characteristically high conductivity and have a high power factor as thermoelectric elements. This result has introduced the possibility of thermoelectric devices that use molecular conductors. BTBT is formed from the benzothiophene skeleton (Scheme 1b), in which the HOMO is largely localized on the sulfur atoms. This property is common to DY units such as TTF. Therefore, we have focused on the benzothiophene skeleton to develop new RC salts. Recently, we developed a 2D Mott insulator $\theta$-(BEDT-BDT) $\mathrm{PF}_{6}$, where BEDT-BDT is benzo[1,2-g:4,5-g']bis(thieno[2,3-b][1,4]dithiin), and reported its structural and physical properties [12]. Because this material remains paramagnetic even at $2 \mathrm{~K}$, the ground state may be a quantum spin liquid. BEDT-BDT is a molecule in which an ethylenedithio group is introduced at the molecular terminal of benzodithiophene, and it may form many 2D RC salts, same as BEDT-TTF. In this paper, we report the structural and physical properties of the new RC salt (BEDT-BDT) $A_{s} F_{6}$. In particular, we describe the correlation between the dihedral angle and transfer integral in the $\theta$-mode arrangement, and discuss the characteristics of the molecular orbitals that are peculiar to BEDT-BDT, which are not found in BEDT-TTF.

\section{Experimental Methods}

\subsection{Electrocrystallization}

BEDT-BDT was synthesized according to a previous study [13]. Briefly, $4-5 \mathrm{mg}$ of BEDT-BDT and $20-25 \mathrm{mg}$ of $(\mathrm{n}-\mathrm{Bu})_{4} \mathrm{NAsF}_{6}$ were dissolved in distilled chlorobenzene $(15 \mathrm{~mL})$. Initially, a current of $0.1 \mu \mathrm{A}$ was applied. The current was increased to $0.5 \mu \mathrm{A}$ over a month. Very thin, reddish-blown, plate-like crystals were obtained. 


\subsection{X-ray Diffraction and Structural Analysis}

X-ray diffraction data of the crystals were collected at 270 and $93 \mathrm{~K}$ using a Rigaku Mercury 70 diffractometer and multilayer mirror monochromated Mo-K $\alpha$ radiation $(\lambda=0.71075 \AA)$. The crystal structures were solved using a direct method (SIR2014) [14] and refined by full-matrix least squares on $F^{2}$ (SHELXL-2014/3) [15].

\subsection{Calculation of Transfer Integrals and Fermi Surfaces}

The intermolecular transfer integrals were estimated using the Amsterdam Density Functional (ADF) program at the PW91/TZP level [16]. Using these transfer integrals, the Fermi surfaces were calculated in the tight-binding approximation [17].

\subsection{Resistivity Measurements}

Transport measurements were performed on single crystals. Gold wires $(10 \mu \mathrm{M}$ in diameter) were attached to the conducting plane with carbon paste. The temperature dependence of the in-plane resistivity was measured using the conventional four-probe DC technique using a Keithley 6221/2182A Delta Mode System.

\subsection{Magnetic-Susceptibility Measurements}

Magnetic susceptibility was measured using a commercial superconducting quantum interface device magnetometer (Quantum Design, MPMS-XL5HG) in the temperature and magnetic-field range of $2-300 \mathrm{~K}$ and $-5 \leq H \leq 5 \mathrm{~T}$, respectively. The measurements were performed with polycrystalline samples $(\sim 3.32 \mathrm{mg})$. The background was subtracted from the data.

\section{Results and Discussion}

Figure 1 shows the crystal structure of the (BEDT-BDT)AsF 6 . Half of the BEDT$\mathrm{BDT}$ and $\mathrm{AsF}_{6}$ anion are crystallographically independent. Because the donor: anion composition ratio was 1:1, the $\mathrm{AsF}_{6}$ salt is considered to be a half-filled Mott insulator. The crystal system was monoclinic and the space group was $C 2 / \mathrm{C}$. Since the $\mathrm{Z}$ value is 4 , there are four molecules each of BEDT-BDT and $\mathrm{AsF}_{6}$ in the unit cell. The $\mathrm{AsF}_{6}$ salt has the same structure as the $\mathrm{PF}_{6}$ salt reported in a previous study [12]; that is, the BEDT-BDT molecules form alternate layers and are arranged in $\theta$-mode packing. However, the anion molecule $\mathrm{AsF}_{6}$ is disordered. As the temperature factor of the fluorine atoms is large, we analyzed the crystallographic data obtained at $270 \mathrm{~K}$, assuming the temperature factor of the fluorine atoms to be isotropic. The BEDT-BDT molecules form a tilted uniform stack along the $b$-axis. The interplanar distance is $3.955 \AA$, which is slightly larger than that in the $\mathrm{PF}_{6}$ salt. There are many short contacts between the BEDT-BDT molecules. S ...S short

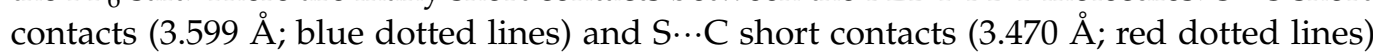
exist between the donor stacks (Figure 1a), whereas S...H short contacts $(2.812 \AA$; green dotted lines) exist within the donor stack (Figure 1b). The intermolecular transfer integrals are $t_{1}=-27 \mathrm{meV}$ and $t_{2}=22 \mathrm{meV}$. The value of $t_{1}$ is slightly smaller than that of the $\mathrm{PF}_{6}$ salt. This is consistent with the increased interplanar distance caused by the larger size of the anion. There is no structural phase transition down to $93 \mathrm{~K}$. The anion molecules are disordered even at $93 \mathrm{~K}$; however, the fluorine atoms could be analyzed as anisotropic (Table S1, see Supporting Information). 
(a)

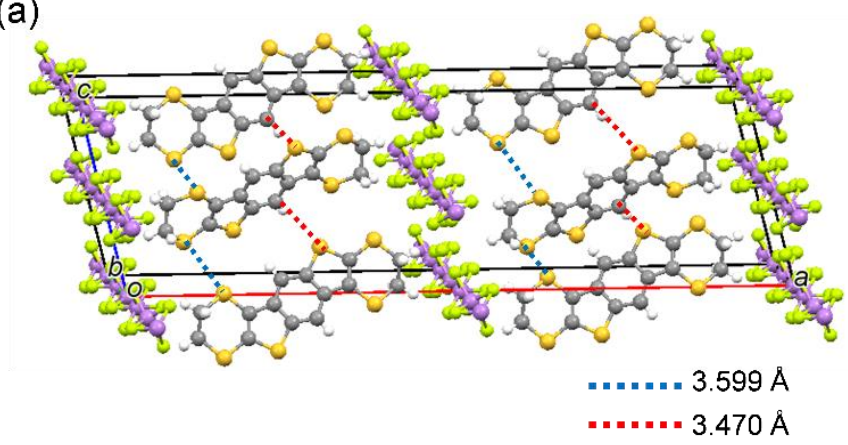

(b)

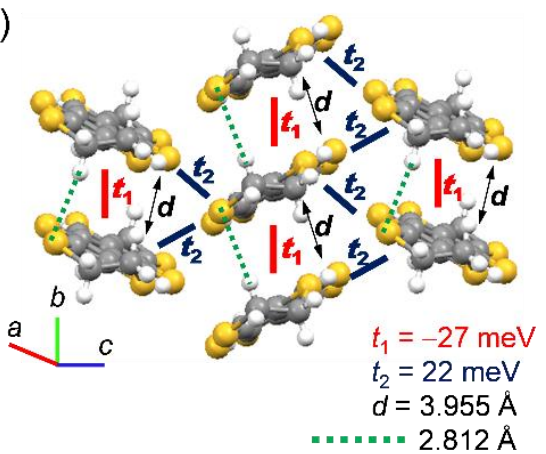

Figure 1. Crystal structures of (BEDT-BDT) $\mathrm{AsF}_{6}$ at $270 \mathrm{~K}$. (a) Molecular arrangements of the donor and anion layers. (b) Donor arrangement in $\theta$-(BEDT-BDT) $\mathrm{AsF}_{6}$ (the solid lines show the intermolecular transfer integrals; the AsF $\mathrm{A}_{6}$ anions are omitted for clarity).

Figure 2 shows the temperature dependence of the resistivity. The resistivity at $298 \mathrm{~K}$ is $3.4 \Omega \mathrm{cm}$. Below $298 \mathrm{~K}$, the resistivity gradually increases, and the sample exhibits semiconducting behavior (Figure 2a). The activation energy $E_{\mathrm{A}}$ is estimated to be $91 \mathrm{meV}$ (Figure $2 \mathrm{~b}$ ). These values are not significantly different from those of the $\mathrm{PF}_{6}$ salt $\left(E_{\mathrm{A}}=87 \mathrm{meV}\right)$. The difference in the temperature-dependent behavior of the resistivity is likely due to the difference in the measurement method (the $\mathrm{PF}_{6}$ salt was measured using the two-terminal method). These results also support the conclusion that the $\mathrm{AsF}_{6}$ salt is a Mott insulator with strong electron correlation.
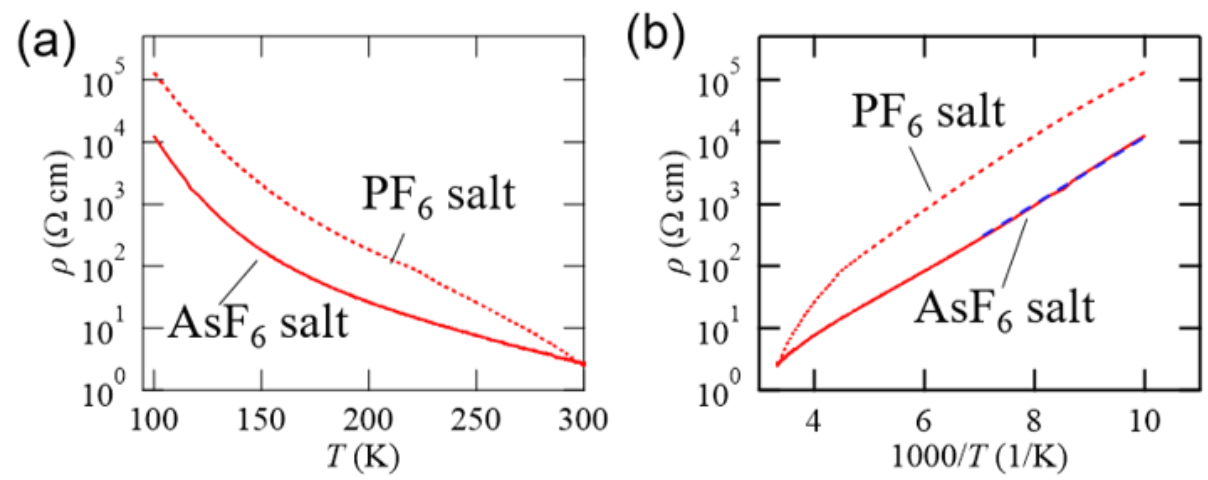

Figure 2. (a) Temperature dependence of the electrical resistivity $\rho$. (b) Arrhenius plot. The red dashed lines are the results for the $\mathrm{PF}_{6}$ salt, which are taken from ref. [12]. The blue dashed line exhibits the fitting curve to estimate an activation energy.

The temperature dependence of the static magnetic susceptibility $(\chi)$ is shown in Figure 3a. The magnitude of $\chi$ is $3.96 \times 10^{-4} \mathrm{emu} / \mathrm{mol}$ at $300 \mathrm{~K}$, which is of the same order as that for typical molecular conductors. The temperature dependence of $\chi$ seems to follow the Curie-Weiss law $\chi=C\left(T-\theta_{\mathrm{W}}\right)$ for a wide temperature range above $\sim 25 \mathrm{~K}$. The $1 / \chi$ plot provides a Curie constant $C=0.14 \mathrm{emu} \mathrm{K} / \mathrm{mol}$ and a Weiss temperature $\theta_{\mathrm{W}}=-5.97 \mathrm{~K}$, respectively (Figure $3 \mathrm{~b}$ ). The spin concentration calculated from $C$ is $37.3 \%$ of the total spin localized at each BEDT-BDT site. Below $25 \mathrm{~K}$, the spins change from a classical-property state to a quantum-property state. Therefore, the behavior of the magnetic susceptibility data follow the Heisenberg model in place of the Curie-Weiss law. It should be noted that the $\mathrm{PF}_{6}$ salt from previous studies showed good agreement with the $2 \mathrm{D}$ triangular model [12], while the $\mathrm{AsF}_{6}$ salt is consistent with the $1 \mathrm{D}$ chain model with the exchange coupling $J=7.4 \mathrm{~K}$ [18]. (Figure S3, see Supporting Information). 
(a)

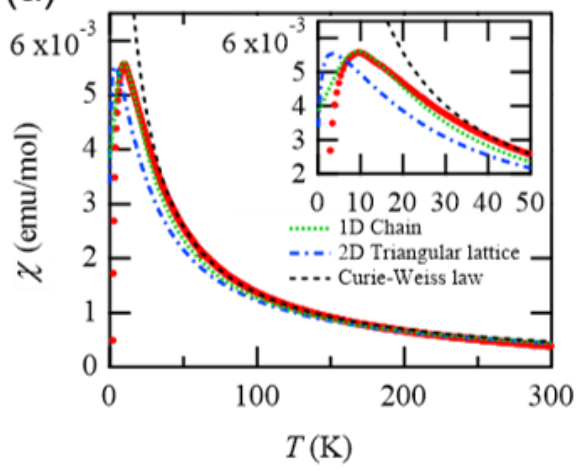

(b)

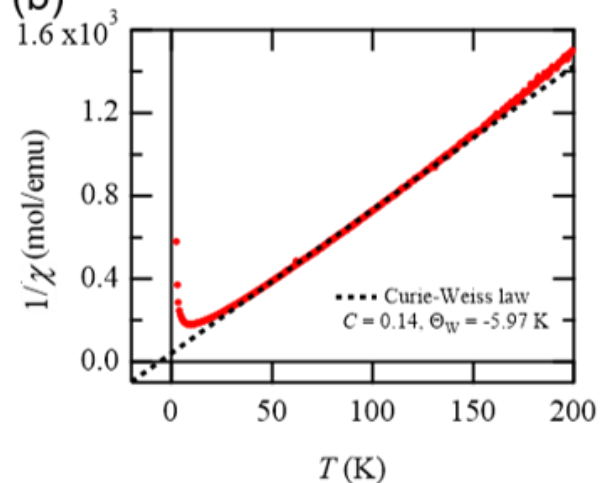

Figure 3. Temperature dependence of (a) magnetic susceptibility. The blue, green, and black dotted lines represent the fitting to the 2D triangular model, 1D chain model, and Curie-Weiss law, respectively. (b) $1 / \chi$ plot.

The different behaviors observed at low temperature for the $\mathrm{PF}_{6}$ and $\mathrm{AsF}_{6}$ salts are discussed with the Fermi surface calculations in the next paragraph.

Figure 4 shows temperature dependence of the calculated Fermi surface. In this study, we performed a structural analysis of the $\mathrm{PF}_{6}$ salt at $93 \mathrm{~K}$ and calculated the Fermi surface. The $\mathrm{PF}_{6}$ salt has almost the same band structure at both 273 and $93 \mathrm{~K}$, and the Fermi surface forms a type of $2 \mathrm{D}$ cylinder. At $270 \mathrm{~K}$, the $\mathrm{AsF}_{6}$ salt has a Fermi surface similar to that of the $\mathrm{PF}_{6}$ salt. However, at $93 \mathrm{~K}$, the band structure changes and the cylinder-type Fermi surface is significantly distorted. This is because the transfer integral $t_{1}$ in the stack direction becomes small. The temperature dependence of the Fermi surface supports the assumption that the $1 \mathrm{D}$ chain model is more appropriate that the $2 \mathrm{D}$ triangular model in the low-temperature region of magnetic susceptibility. However, this change in the Fermi surface of the $\mathrm{AsF}_{6}$ salt is not due to a structural phase transition (Table S1, see Supporting Information).

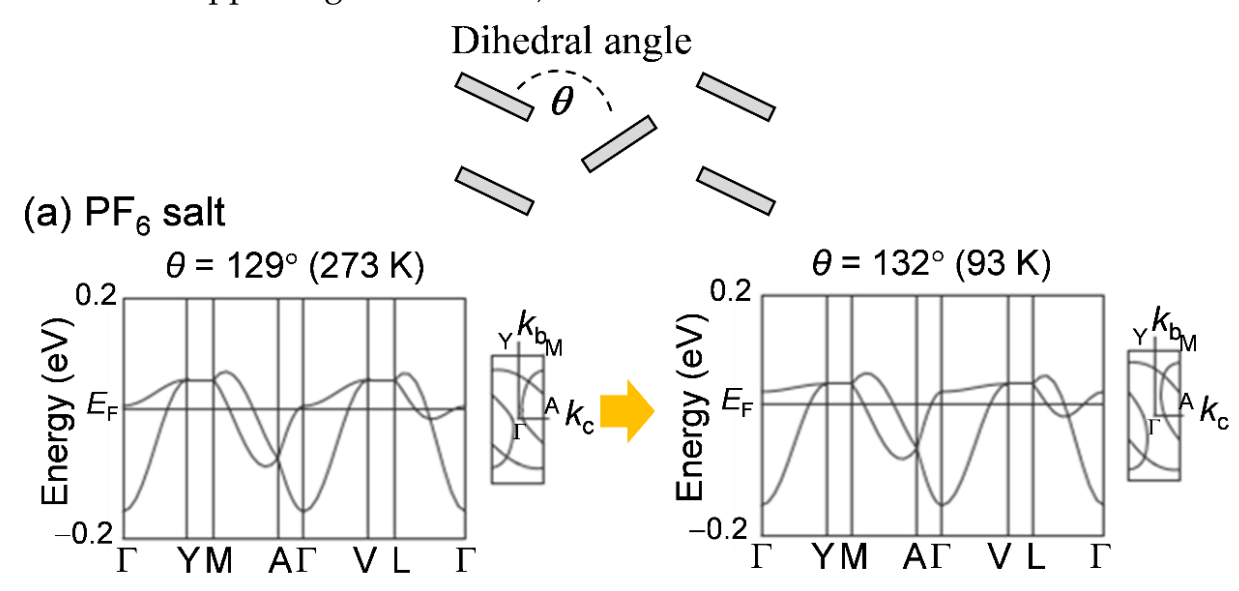

(b) $\mathrm{AsF}_{6}$ salt
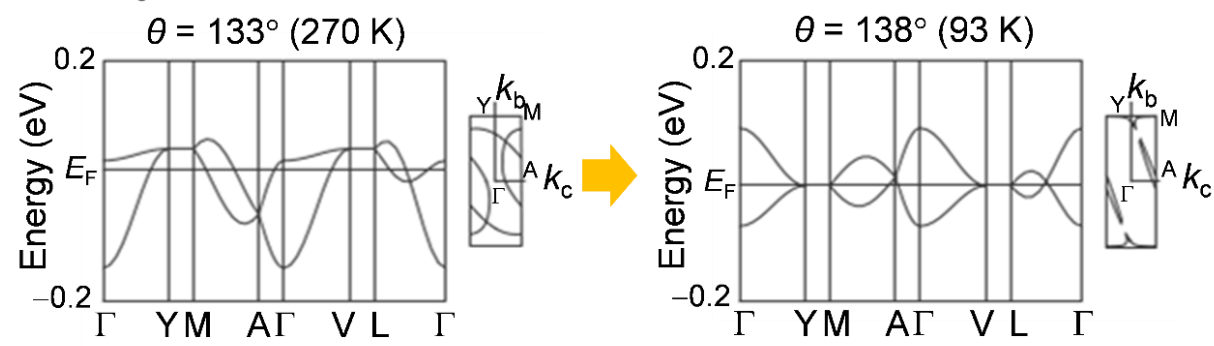

Figure 4. Temperature dependence of the band dispersions and Fermi surfaces of the (a) $\mathrm{PF}_{6}$ and (b) $\mathrm{AsF}_{6}$ salts. 
Table 1 shows the HOMO energy, transfer integral, interplanar distance, and dihedral angle at each temperature. The value of the HOMO energy in one molecule of BEDTBDT is $\sim-4.5 \mathrm{eV}$. This value does not depend greatly on either the difference between the counter anions or the temperature. Therefore, it is possible to compare the transfer integrals. The value of $t_{2}$ does not change between stacks; however, the value of $\left|t_{1}\right|$ within a stack is extremely small at $93 \mathrm{~K}$ for the $\mathrm{AsF}_{6}$ salt. This change in $\left|t_{1}\right|$ depends more on the dihedral angle $\theta$ between the stacks than on the interplanar distance $d$ between the donor molecules in the stack. Specifically, we confirm that $\left|t_{1}\right|$ tends to decrease as $\theta$ increases. Studies using molecular-orbital calculations have reported that the value of $\left|t_{1}\right|$ in the stack changes depending on the dihedral angle $\theta$ in a $\theta$-type molecular arrangement [19]. Typical examples with $\theta$-type arrangements are the BEDT-TTF salts $[20,21]$. In these BEDTTTF salts, $\left|t_{1}\right|$ is smaller than $\left|t_{2}\right|$. This is because BEDT-TTF has multiple sulfur atoms aligned around the molecule. By contrast, the $\theta$-type BEDT-BDT salts have $\left|t_{1}\right|>\left|t_{2}\right|$ as shown in Table 1.

Table 1. Temperature dependence of the HOMO energy, transfer integrals $\left(t_{1}\right.$ and $\left.t_{2}\right)$, interplanar distance $d$, and dihedral angle $\theta$ of the BEDT-BDT salts.

\begin{tabular}{ccccccc}
\hline & $\boldsymbol{T}(\mathbf{K})$ & HOMO $(\mathbf{e V})$ & $\boldsymbol{t}_{\mathbf{1}}(\mathbf{m e V})$ & $\boldsymbol{t}_{\mathbf{2}}(\mathbf{m e V})$ & $\boldsymbol{d}(\AA)$ & $\boldsymbol{\theta}\left({ }^{\circ}\right)$ \\
\hline$($ BEDT-BDT)PF & 273 & -4.50 & -33 & 22 & 3.947 & 128.6 \\
- & 93 & -4.56 & -27 & 23 & 3.901 & 131.5 \\
$\left(\mathrm{BEDT}_{6}\right.$ & 270 & -4.58 & -27 & 22 & 3.955 & 133.1 \\
- & 93 & -4.52 & 3.6 & 20 & 3.910 & 137.8 \\
\hline
\end{tabular}

Figure 5 shows the dihedral-angle dependence of the transfer integrals of the BEDTBDT and BEDT-TTF salts. As illustrated in Figure 5a, the overlap between molecules in the stack is generally expected to increase as $\theta$ increases; thus, $t_{1}$ increases. In the $\theta$-type BEDT-TTF salts, $\theta$ changes depending on the size of the counter anion [20]. As $\theta$ increases, the absolute value of $t_{1},\left|t_{1}\right|$, increases linearly (Figure 5b). By contrast, in the BEDT-BDT salts, $\left|t_{1}\right|$ decreases as $\theta$ increases. The change is not a monotonic linear change but exhibits a sharp curve with a local maximum at the point $\theta=128^{\circ}$ (the $\mathrm{PF}_{6}$ salt at $273 \mathrm{~K}$ ). The changes in $t_{2}$ are $94<t_{2}<108 \mathrm{meV}$ for the BEDT-TTF salts and $20<t_{2}<23 \mathrm{meV}$ for the BEDT-BDT salts. No significant changes are observed in either salt compared with $t_{1}$. The fact that $t_{1}$ in the stack of the BEDT-BDT salts is sensitive to $\theta$ is due to the shape of the molecular orbital.

(a)

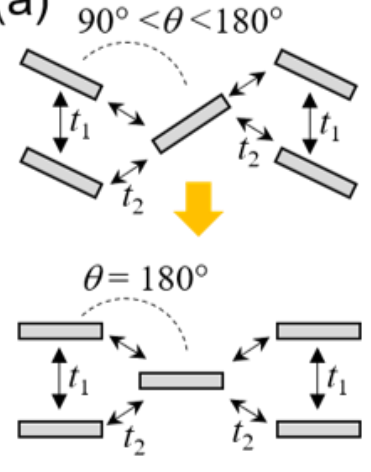

(b)

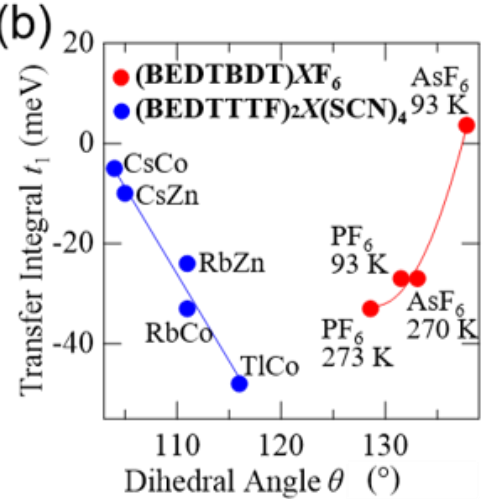

(c)

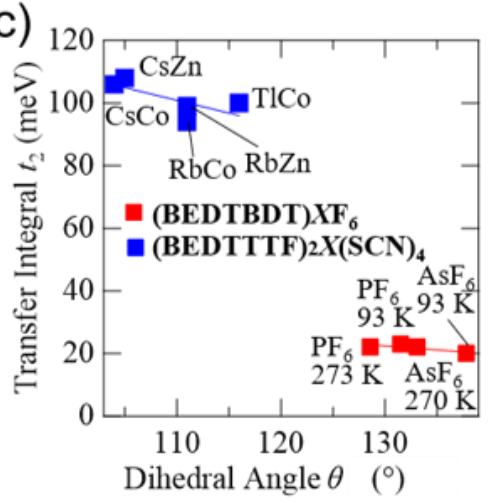

Figure 5. Dihedral-angle dependence of the (a) $\theta$-type molecular arrangements and transfer integrals (b) $t_{1}$ and (c) $t_{2}$.

Figure 6 shows the phase (sign) symmetry of the HOMO for BEDT-TTF and BEDT-BDT. The HOMO phase of BEDT-TTF remains the same under the rotation operation. The shapes and phases of the HOMO are symmetrical with respect to both the long and short molecular axes. Therefore, the HOMO shape and phase have high symmetry. Regarding the HOMO, we focus on the sulfur atoms with $d$-orbitals that make substantial contributions to the 
intermolecular interactions. The HOMO of BEDT-TTF is localized at each sulfur atom, and the HOMO coefficient is particularly large at the sulfur atoms in the TTF skeleton [12]. Figure $6 \mathrm{c}$ demonstrates that the HOMO on the sulfur atoms at the TTF sites is larger than that on the carbon atoms. The HOMO above the sulfur atoms has the same phase, and the dominant HOMO phase in the molecular plane of BEDT-TTF is determined by the phase of the HOMO on the sulfur atoms in the TTF moiety. This property has been reported in previous studies [22,23]. This dominant HOMO phase is reversed with respect to the long plane of the molecule. For example, if the upper side of the molecular plane has a positive phase, the lower HOMO phase is negative. This is a characteristic of BEDT-TTF. However, the HOMO of BEDT-BDT has lower symmetry than that of BEDT-TTF because the only symmetry element is the rotation operation. The phase of the HOMO is inverted on the left and right sides of the central benzene ring. Because the positive and negative phases exist equally, there is no dominant phase throughout the molecular plane. That is, the sign of the HOMO is neutral on both the upper and lower sides of the molecular plane.

(a) the molecular
short axis

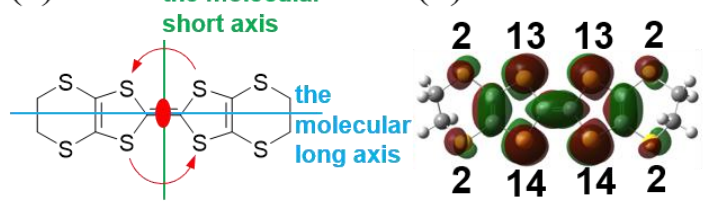

(d)

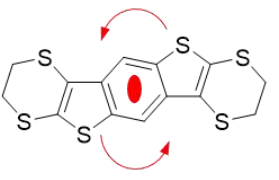

(b)

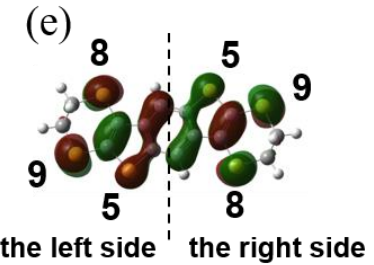

(c)

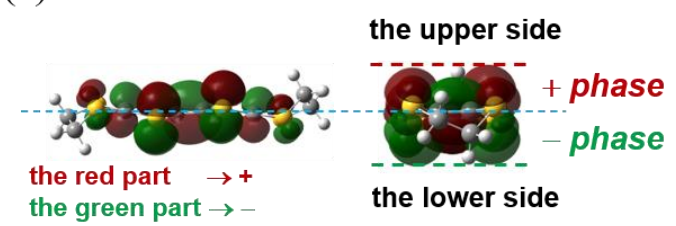

(f)

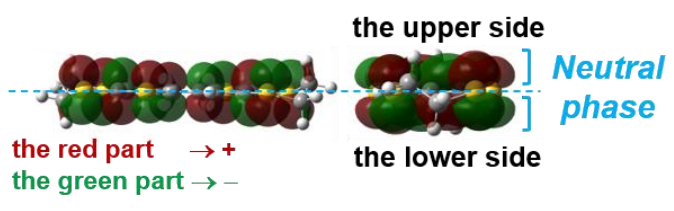

Figure 6. (a) Molecular structure and symmetry and $(\mathbf{b}, \mathbf{c})$ the HOMO distribution and shape of BEDT-TTF. The numbers represent the contributions (\%) of the sulfur atoms to HOMO. (d) Molecular structure and symmetry and (e,f) the HOMO distribution and shape of BEDT-BDT.

The sign of $t_{1}$ in the BEDT-TTF salts switches periodically between positive and negative at intervals of $\theta=60^{\circ}$ due to the phase relationship of the HOMO [19]. By contrast, the sign of $t_{1}$ in the BEDT-BDT salts is much more sensitive to the dihedral angle $\theta$ than the sign of $t_{1}$ of the BEDT-TTF salts. The sign changes from negative to positive at intervals of $\theta=10^{\circ}$. That is, there is a state in which $t_{1}=0$ (the transfer integrals disappear) with only a slight change in the dihedral angle. This has a significant effect on the electronic structure of the BEDT-BDT-based RC salts. In fact, even a small variation such as thermal shrinkage due to a decrease in the temperature changes the dimensionality of the Fermi surface (Figure $4 \mathrm{~b}$ ). A slight change in the dihedral angle causes a significant change in the electronic structure, which is a characteristic of RC salts using BEDT-BDT because the HOMO does not have a dominant sign throughout the molecule. In other words, the BEDT-BDT salt is considered to be more pressure sensitive than conventional molecular conductors. This property is expected to lead to the development of new transistors whose conduction path changes due to the anisotropic pressure effects from the substrate [24-26].

\section{Conclusions}

In conclusion, we investigated the structural and physical properties of the new RC salt (BEDT-BDT) $\mathrm{AsF}_{6}$. Moreover, we successfully performed the structural analysis of (BEDT-BDT)PF $\mathrm{P}_{6}$ at $93 \mathrm{~K}$. The $\mathrm{AsF}_{6}$ salt is a $2 \mathrm{D}$ Mott insulator with the same structure as the $\mathrm{PF}_{6}$ salt. At temperatures below $300 \mathrm{~K}$, the resistivity gradually increases and the salt exhibits semiconductor behavior with an activation energy of $91 \mathrm{meV}$. The static magnetic susceptibility essentially follows Curie-Weiss law but is consistent with a 1D chain model 
with $J=7.4 \mathrm{~K}$ below $25 \mathrm{~K}$. When the temperature decreased from 270 to $93 \mathrm{~K}$, the band dispersion and Fermi surface of the $\mathrm{AsF}_{6}$ salt changed significantly. This is because the dihedral angle between the BEDT-BDT molecules increased due to thermal shrinkage. In a $\theta$-type arrangement, the transfer integrals change depending on the dihedral angle, but the BEDT-BDT salts exhibit larger dihedral-angle dependence than the BEDT-TTF salts. This is because BEDT-BDT has only rotational symmetry and there is no dominant HOMO phase throughout the molecule. It is characteristic of the BEDT-BDT salts that the transfer integrals are sensitive to slight differences in the overlap between the molecules, and the dimensionality of the electronic structure changes more sensitively than that of conventional $\theta$-type BEDT-TTF conductors.

Supplementary Materials: The following are available online at https:/ /www.mdpi.com/article/ 10.3390/cryst11080868/s1, Figure S1: (a) Definition of label at each bond of BEDT-BDS molecule. (b) Relationship between bond length and molecular valence, Figure S2: (a) A plane definition of the BEDT-BDT and (b) a dihedral angle $\theta$ between the planes formed by two BEDT-BDT molecules on adjacent stacks, Figure S3: Exchange coupling $J$ dependence of the calculation results for (a) 2D triangular lattice and (b) $1 \mathrm{D}$ chain model. Table S1: Crystallographic data of $\theta$-(BEDT-BDT)AsF $\mathrm{F}_{6}$ and

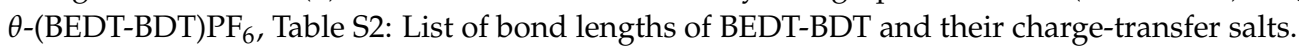

Author Contributions: T.K. conceived and designed the experiments; T.K. developed all the molecular conductors; T.K., S.S., and T.S. participated in all the physical measurements; T.H. and K.T. (Keishiro Tahara) performed the DFT calculation. T.K., K.K., K.T. (Kazuo Takimiya), and J.-i.Y. wrote and/or reviewed the paper with contributions from all authors. All authors have read and agreed to the published version of the manuscript.

Funding: This research was partially supported by JSPS KAKENHI (Grant numbers: JP20K15356), the Kansai Research Foundation for Technology Promotion, Foundation of Kinoshita Memorial Enterprise, Masuyakinen basic research foundation, THE EBARA HATAKEYAMA MEMORIAL FOUNDATION (a public interest incorporated foundation), and Kato Foundation for Promotion of Science.

Data Availability Statement: Not applicable.

Conflicts of Interest: The authors declare no conflict of interest.

\section{References}

1. Ishiguro, T.; Yamaji, K.; Saito, G. Organic Superconductors, 2nd ed.; Springer: Berlin/Heidelberg, Germany, 1998.

2. Mori, T. Electronic Properties of Organic Conductors; Springer: Tokyo, Japan, 2016.

3. Mori, T. Principles that Govern Electronic Transport in Organic Conductors and Transistors. Bull. Chem. Soc. Jpn. 2016, 89, 973-986. [CrossRef]

4. Morawitz, H. Orientational Peierls Transition in Quasi One-Dimensional Organic Solids. Phys. Rev. Lett. 1975, 34, 1096-1099. [CrossRef]

5. Mori, H. Materials Viewpoint of Organic Superconductors. J. Phys. Soc. Jpn. 2006, 75, 051003. [CrossRef]

6. Saito, G.; Yoshida, Y. Development of Conductive Organic Molecular Assemblies: Organic Metals, Superconductors, and Exotic Functional Materials. Bull. Chem. Soc. Jpn. 2007, 80, 1-137. [CrossRef]

7. Shirahata, T.; Kohno, S.; Furuta, K.; Oka, Y.; Misaki, Y. Synthesis of New Electron Donor ClMe 3 -TTP: Structures and Properties of $\left(\mathrm{ClMe}_{3}-\mathrm{TTP}\right)_{3} \mathrm{X}\left(\mathrm{X}=\mathrm{PF}_{6}\right.$ and $\left.\mathrm{AsF}_{6}\right)$. Bull. Chem. Soc. Jpn. 2015, 88, 1086-1092. [CrossRef]

8. Kadoya, T.; Ashizawa, M.; Higashino, T.; Kawamoto, T.; Kumeta, S.; Matsumoto, H.; Mori, T. A Highly Conducting Organic Metal Derived from an Organic-Transistor Material: Benzothienobenzothiophene. Phys. Chem. Chem. Phys. 2013, 15, 17818-17822. [CrossRef] [PubMed]

9. Kiyota, Y.; Kadoya, T.; Yamamoto, K.; Iijima, K.; Higashino, T.; Kawamoto, T.; Takimiya, K.; Mori, T. BenzothienobenzothiopheneBased Molecular Conductors: High Conductivity, Large Thermoelectric Power Factor, and One-Dimensional Instability. J. Am. Chem. Soc. 2016, 138, 3920-3925. [CrossRef]

10. Higashino, T.; Kadoya, T.; Kumeta, S.; Kurata, K.; Kawamoto, T.; Mori, T. An Organic Metal Derived from a Selenium Analogue of Benzothienobenzothiophene. Eur. J. Inorg. Chem. 2014, 24, 3895-3898. [CrossRef]

11. Kadoya, T.; Oki, R.; Kiyota, Y.; Koyama, Y.; Higashino, T.; Kubo, K.; Mori, T.; Yamada, J. Transport Properties of Molecular Conductors (BSBS) ${ }_{2} \mathrm{XF}_{6}(\mathrm{X}=\mathrm{As}, \mathrm{Sb}$, and Ta): Investigation of Intermolecular Transfer Integrals in the Radical-Cationic State of BTBT-Type Semiconductors. J. Phys. Chem. C 2019, 123, 5216-5222. [CrossRef]

12. Kadoya, T.; Sugiura, S.; Tahara, K.; Higashino, T.; Kubo, K.; Sasaki, T.; Takimiya, K.; Yamada, J. Two-Dimensional Radical-Cationic Mott Insulator Based on an Electron Donor Containing Neither Tetrathiafulvalene nor Tetrathiapentalene Skeleton. CrystEngComm 2020, 22, 5949-5953. [CrossRef] 
13. Wang, C.; Nakamura, H.; Sugino, H.; Takimiya, K. Thiacycle-fused benzo[1,2-b:4,5- $b^{\prime}$ ]dithiophenes (BDTs): Synthesis, Packing, Molecular Orientation and Semiconducting Properties. J. Mater. Chem. C 2018, 6, 3604-3612. [CrossRef]

14. Burla, M.C.; Caliandro, R.; Carrozzini, B.; Cascarano, G.L.; Cuocci, C.; Giacovazzo, C.C.; Mallamo, M.; Mazzone, A.; Polidori, G. Crystal Structure Determination and Refinement via SIR2014. J. Appl. Crystallogr. 2015, 48, 306-309. [CrossRef]

15. Sheldrick, G.M. A short history of SHELX. Acta. Cryst. A 2008, 64, 112-122. [CrossRef] [PubMed]

16. ADF: Powerful DFT Code for Modeling Molecules; Scientific Computing and Modeling: Amsterdam, The Netherlands. Available online: http:/ / www.scm.com/ADF/ (accessed on 30 June 2021).

17. Mori, T.; Kobayashi, A.; Sasaki, Y.; Kobayashi, H.; Saito, G.; Inokuchi, H. The Intermolecular Interaction of Tetrathiafulvalene and Bis(ethylenedithio)tetrathiafulvalene in Organic Metals. Calculation of Orbital Overlaps and Models of Energy-band Structures. Bull. Chem. Soc. Jpn. 1984, 57, 627-633. [CrossRef]

18. Ami, T.; Crawford, M.K.; Harlow, R.L.; Wang, Z.R.; Johnston, D.C.; Huang, Q.; Erwin, R.W. Magnetic Susceptibility and Low-Temperature Structure of the Linear Chain Cuprate $\mathrm{Sr}_{2} \mathrm{CuO}_{3}$. Phys. Rev. B 1995, 51, 5994. [CrossRef]

19. Kojima, H.; Mori, T. Dihedral Angle Dependence of Transfer Integrals in Organic Semiconductors with Herringbone Structures. Bull. Chem. Soc. Jpn. 2011, 84, 1049-1056. [CrossRef]

20. Mori, H.; Tanaka, S.; Mori, T.; Kobayashi, A.; Kobayashi, H. Crystal Structure and Physical Properties of M $=\mathrm{Rb}$ and Tl Salts (BEDT-TTF)2MM'(SCN)4 [M' = Co, Zn]. Bull. Chem. Soc. Jpn. 1998, 71, 797-806. [CrossRef]

21. Mori, H.; Tanaka, S.; Mori, T. Systematic study of the electronic state in $\theta$-type BEDT-TTF organic conductors by changing the electronic correlation. Phys. Rev. B 1998, 57, 12023. [CrossRef]

22. Mori, T. Structural Genealogy of BEDT-TTF-Based Organic Conductors I. Parallel Molecules: $\beta$ and $\beta^{\prime \prime}$ Phases. Bull. Chem. Soc. Jpn. 1998, 71, 2509-2526. [CrossRef]

23. Mori, T.; Mori, H.; Tanaka, S. Structural Genealogy of BEDT-TTF-Based Organic Conductors II. Inclined Molecules: $\theta, \alpha$, and $\kappa$ Phases. Bull. Chem. Soc. Jpn. 1999, 72, 179-197. [CrossRef]

24. Kawasugi, Y.; Yamamoto, H.M.; Hosoda, M.; Tajima, N.; Fukunaga, T.; Tsukagoshi, K.; Kato, R. Strain-Induced Superconductor/Insulator Transition and Field Effect in a Thin Single Crystal of Molecular Conductor. Appl. Phys. Lett. 2008, $92,243508$. [CrossRef]

25. Yamamoto, H.M.; Nakano, M.; Suda, M.; Iwasa, Y.; Kawasaki, M.; Kato, R. A Strained Organic Field-Effect Transistor with a Gate-Tunable Superconducting Channel. Nat. Commun. 2013, 4, 2379. [CrossRef] [PubMed]

26. Suda, M.; Kawasugi, Y.; Minari, T.; Tsukagoshi, K.; Kato, R.; Yamamoto, H.M. Strain-Tunable Superconducting Field-Effect Transistor with an Organic Strongly-Correlated Electron System. Adv. Mater. 2014, 26, 3490-3495. [CrossRef] [PubMed] 